“ (C) 2018 IEEE. Personal use of this material is permitted. Permission from IEEE must be obtained for all other uses, in any current or future media, including

reprinting/republishing this material for advertising or promotional purposes, creating new collective works, for resale or redistribution to servers or lists, or reuse of any copyrighted component of this work in other works." 


\title{
Adaptive Sampling for Spatial Prediction in Environmental Monitoring using Wireless Sensor Networks: A Review
}

\author{
Linh Nguyen, Nalika Ulapane and Jaime Valls Miro \\ Centre for Autonomous Systems, Faculty of Engineering and Information Technology \\ University of Technology Sydney, Australia \\ Email: \{vanlinh.nguyen, nalika.ulapane, jaime.vallsmiro\}@uts.edu.au
}

\begin{abstract}
The paper presents a review of the spatial prediction problem in the environmental monitoring applications by utilizing stationary and mobile robotic wireless sensor networks. First, the problem of selecting the best subset of stationary wireless sensors monitoring environmental phenomena in terms of sensing quality is surveyed. Then, predictive inference approaches and sampling algorithms for mobile sensing agents to optimally observe spatially physical processes in the existing works are analysed.
\end{abstract}

\section{INTRODUCTION}

Environmental issues such as pollution of land, water and air, drastic climate change, natural disasters, and resource depletion have topped the agenda in recent years. These are crucial not only to governments but also to environmental scientists. Therefore, it would be prudent to monitor spatially correlated environmental phenomena so as to ameliorate the knowledge and understanding of their economic, environmental, and health impacts and implications. For instance, determining distributional patterns of ecological phenomena can be utilized to design resource-specific exploitation plans [1]. Better understanding of physics underlying occurrence process of earthquakes [2] can generate early vital hazard warnings to society. Results from observing sulphur dioxide $\mathrm{SO}_{2}$ in the air [3], which may affect the respiratory system, should be given to alert community council to reduce burning of sulphur-containing fuels in factories. In the agricultural field, monitoring nitrogen density in the soil can be used to regulate farm inputs that leads to mitigation of environmental pollution due to over-application of nitrogen fertilizer [4]. In addition to concerns mentioned above, there is required ubiquitous observations of temperature, humidity, rainfall, soil ingredients, and mono-nitrogen oxides $N O_{x}$ in natural and built-up habitats [5], [6]. Nonetheless, since environmental measurements are a single scalar quantity that is only locally valid, it is required to make predictions about the process at unmeasured locations by using observations [7]-[9]. Therefore, there are currently strong motivations to monitor, model and predict the environmental field of interest that is often represented as complex phenomena. More particularly, if sensing equipment is effectively used in monitoring, modelling and predicting the

This work was supported by the Critical Pipes Project funded by Sydney Water Cooperation and University of Technology Sydney, Australia. spatial phenomena, which is referred to as adaptive sampling, results from visually representing the physical field are useful in making decisions regarding environmental issues.

The typical task of wireless sensor networks (WSN) [10] consists of gathering measurements of a spatial field over a region of interest. However, for instance in a stationary wireless sensor network (SWSN), multiple wireless sensor nodes co-located within the vicinity of a phenomenon in a dense SWSN may generate similar data samples. This over-sampling problem has the potential to cause a sizeable redundancy in sensed data, data collection and analysis of long-term monitoring to be very expensive. Consequently, it is crucial to select the most informative subset of stationary wireless sensor nodes out of all potential ones, which should participate in the sensing task. The selection procedure is known as a sensor selection problem in which the resulting prediction is required to conform requirement of the highly sensing quality in realistic applications.

Nevertheless, if the networks of stationary wireless sensors are deployed in a changing environment, the stationary wireless sensor locations selected by a sensor selection algorithm are no longer the most informative over time. Hence, a wireless sensor network incorporating mobile robotic platforms is desirable. With a set of networked mobile sensor nodes, the mobile robotic wireless sensor networks (MRWSNs) are capable of providing services required not only for monitoring but also for exploring the environment. Effectively utilizing the MRWSNs to observe and predict the environmental fields is widely considered as a sensor placement problem.

This paper is to summarize all approaches in literature proposed to address the problems of sensor selection and sensor placement.

\section{Sensor Selection in SWSNs}

\section{A. Criteria for Sensor Selection}

In statistics, selecting observations has been considered as an experimental design problem [11]-[14]. The design objective is to derive the deployment of sensing devices by the use of model uncertainty, which could be formulated by complicated statistical techniques. The optimality criteria were constructed based upon the properties of the inverse moment matrix. For instance, D-optimality considers the determinant 
[15], A-optimality examines the trace and E-optimality calculates the maximum eigenvalue [12].

Recently, research attention to the sensor selection has concentrated on selecting observations in order to maximize the quality of parameter estimation [16]-[18] with a special focus on linear models that are often coupled with a stochastic measurement error term. The sensor selection metric can be formulated in a Bayesian framework [19] in which it is supposed to have the knowledge prior to carrying out the experiments. In equivalent words, combining the prior probability distribution of the parameter space with the observations, the design criteria can be derived. The sensor selection criteria are also defined based upon scalar functions of the Fisher information matrix or the Bayesian Fisher information matrix [18]. However, in the context of spatial prediction, the design objective frequently concerned is the quality of sensing, which is described as the accuracy of prediction or the uncertainty at unobserved locations of interest, after the observations are made. This requirement has been utilized to develop information-theoretic criteria [20]-[24].

\section{B. Algorithms for Sensor Selection}

In terms of sensor selection algorithms, one can simply process all direct enumerations of $\left(\begin{array}{l}n \\ k\end{array}\right)$ possible choices and pick the best subset of $k$ sensors out of $n$ potential ones having the minimal prediction error. It can be seen that this straightforward approach has practical implications depending on the values of $n$ and $k$ and thus motivates more structured methods. In [25], the global optimization techniques such as branch and bound were employed to exactly solve this problem. Nevertheless, since the sensor selection problem, which can be viewed as a combinatorial optimization problem, is NP-hard [26], [27], these accurate approaches are often computationally intensive [28], even with modest values of $n$ and $k$, and not attractive in real world solutions.

In an effort to improve the model parameter estimation, there have been some interesting methods proposed. For instance, in [16], Joshi et al. proposed the heuristic method based on convex optimization [11] for the sensor selection problem. The heuristic approach in [16] utilizes a relaxation technique to convert a discrete optimization problem of sensor selection into a continuous optimization problem. Gupta et al. [29] in their work represented a stochastic sensor selection algorithm that selects sensor locations randomly by the use of a probability distribution. Maximum information in the estimation of the state variables can be obtained by a mixed-integer semi-definite program approach [22]. In [17], a binary particle swarm optimization technique was employed to choose a subset of sensors so that the error in parameter estimation is minimized.

Based on Bayesian experimental design, the informationtheoretic approaches such as entropy [30] or mutual information [31], [32] were proposed to consider the prediction uncertainty of the random variables at unobserved locations in space. The greedy heuristic algorithms based on these information-theoretic models together with Gaussian processes proposed by Cressie [33] can obtain near-optimal solutions for the sensor selection problem. These algorithms were demonstrated in the works [7], [20], [26], [34], [35]. The premise behind the entropy approach is to minimize the uncertainty of conditional entropy of unobserved locations, given observations. Under Gaussian assumption, Ko et al. [26] proposed a greedy suboptimal algorithm by reorganizing the maximization of joint entropy of a chosen set as maximizing the determinant of the covariance matrix of random variables at chosen locations. However, as shown in [36], the entropy method tends to pick locations along the border of interested space causing sensed information waste. To address the drawbacks of the entropy approach, the work in [20] and our previous works in [21], [37] proposed a new method based on the mutual information. In this method a subset $k$ from potential $n$ sensor locations is selected such that the mutual information between the selected subset and the rest of the sensor locations is maximal. In the context of the sensor selection problem, this maximum mutual information is obtained indirectly.

The greedy approximation [38], [39] is a simple, but often used, algorithm for combinatorial optimization problems which can be directly applied in the sensor selection problem. By building up a solution piece by piece, the greedy approximation algorithm may complete the computational tasks in a few seconds. In every iteration, one sensor is chosen and moved to the selected set. Once a sensor is moved to the selected set it is impossible to remove it from the selected set during later iterations. Nevertheless, this prime disadvantage of the algorithm voids the solution to reach optimal values. Another prominent heuristic algorithm to solve combinatorial optimization problem is the genetic algorithm. The genetic algorithm imitates the evolutionary process of nature in which a solution deputizes for the organisms' genetic string. Yao et al. [40] in their work illustrated the use of the genetic algorithm to solve the sensor placement problem. Such an approach, however, can be very expensive for some computational costs when going over a population of individuals [41].

\section{Sensor Placement in MRWSNs}

In the wireless sensor network and robotic research community, mobile robotic sensor nodes have attracted much recent attention due to their vital impact on applications such as surveillance, environmental monitoring, wildlife detection and urban search and rescue operations [42]. The mobile ability of robotics can be utilized to improve performance in WSNs such as node localization, data collection, data aggregation and detection and reaction of failed nodes.

With robots embedded in the sensor network, mobile robotic wireless sensor nodes provide a considerable benefit to connectivity, cost, reliability, and energy efficiency throughout the network as compared with a stationary wireless sensor network [43], [44]. Furthermore, the mobility of wireless sensors allows enhancement of the connectivity in a sparse WSN [45], [46]. However, the most widely utilized advantage of mobile robots in the WSNs is to efficiently improve data collection [47]-[50]. The combination of the new paradigm 
brings in new opportunities to reduce and better distribute the energy usage within the network. Particularly, mobile agents have the potential to decline the number of hops in data transmission, which justifies the reduction of energy used to transmit the data and avoids the funnelling effect in centralized WSNs.

\section{A. Sensor Placement in WSNs}

The term sensor placement has been used in various contexts of the WSNs. For instance, in the work by Fletcher et al. [51], an algorithm named Randomized Robot-assisted Relocation of Static Sensors (R3S2) is proposed to utilize mobile robots for the purpose of servicing the WSNs. Specifically, in R3S2, robots travel around the network to discover sensing holes that are not being covered due to unpredictable node failure, then move redundant sensors to the uncovered area. Similarly to the work [51] in terms of servicing systems, authors in [52] examine robot task allocation and robot task fulfilment in wireless sensor and robot networks. For example, the network will organize a group of robots to achieve a desired goal, while other moving robots will recharge batteries on the nodes in the regions of the network. Moreover, there are algorithms proposed in [53], [54] for placement of relocatable nodes in order to improve network connectivity. Considering a largescale static WSN, [55], [56] propose approaches that also employ mobile robots to detect and report failed sensors and then replace these broken nodes.

\section{B. Sensor Placement for Environmental Monitoring}

In the context of monitoring spatial environmental fields, the sensor placement problem for predicting the spatial phenomena has been investigated, which has considerably contributed to a number of interesting approaches and algorithms. In [57], [58], locational optimization had been proposed in optimizing the mobile sensor network locations with respect to a known event probability density in the spatial environment. However, physical processes are not known a priori, and a density function can be only established when measurements are to be taken. Leonard et al. [59] employed a linear model to predict an ocean field and proposed a performance metric that minimizes uncertainty in a model estimate of the sampled field to derive a parameterized family of paths for the mobile sensor networks. By combining with coverage control [57], Martínez [60] derived a distributed prediction scheme based on a nearest neighbour interpolation approach for field estimation in mobile sensor networks. The primary disadvantage of the linear models in both [59] and [60] is that the model parameters must be known a priori. In terms of the compressive sensing framework, Huang et al. [61] maximized the entropy of next measurements to find the next most informative positions for networked mobile sensors to reconstruct an unknown sensing field. By defining a graph whose vertices and edges are considered as a single robot's visiting locations and moving paths, respectively, a path planning algorithm for a mobile robot was proposed in [62] so as to maximize information gained from measurements of a spatio-temporal phenomenon.
Ouyang et al. [63] utilized a Dirichlet process mixture of Gaussian processes to model the continuous-valued spatial field and then proposed a decentralized multi-robot Bayesoptimal active learning policy for multiple robotic sensors so that the most informative partitions of a non-stationary phenomenon are to be sampled. The policy is then resolved by a greedy algorithm. The works in [64], [65], La et al. used consensus filters to propose a distributed sensor fusion algorithm for multiple mobile sensor nodes to automatically adjust their movements to obtain quasi uniform confidence of estimating and mapping a scalar field. By utilizing a Kalman filter for a downsampled system, [66] has developed the optimal sampling strategies in order to balance the estimation quality and the sensor network lifetime.

In terms of statistics, the spatially environmental phenomena are efficiently and effectively modelled by the Gaussian processes (GPs). Therefore, Suh et al. by their work [67] represented an environmental monitoring navigation strategy for a sensing robot, in which the information gain along the robot's trajectory is maximized. Considering a team of sensing agents, Graham et al. described the random field models by tools from geostatistics, that is Kriging, and proposed to utilize either a known [68] or an unknown [69] covariance function. The sensor network includes static computing nodes and mobile sensing agents taking measurements of a random process. The static nodes compute the gradient of variance and send control commands to robotic sensors. Nevertheless, in [70], maximizing joint entropy of measurements in a distributed fashion was investigated to consider the adaptive sampling paths, where motion coordination was designed based upon Voronoi partitions.

Popa et al. proposed extended Kalman filter [71] and nonlinear extended Kalman filter [72] based adaptive sampling approaches to optimally estimate the parameters of distributed variable field models. These schemes also aim to decline the uncertainty in the knowledge of a linear-in-parameters field distribution (linear model). Choi et al. [73] introduced a Kalman filter based technique to learn the parameters of a physical spatio-temporal process model and then presented criteria to navigate mobile sensors throughout an environment in order to maximize a specified performance. In [74], the authors delineated an objective function of a trajectory optimization problem for robotic wireless sensors as a deterministic optimal control problem. In other words, the objective function is imposed on minimizing the variance of the estimate of the environment, which is eventually resolved by a dynamic programming algorithm. Euler et al. in their work [75] introduced a sampling navigation scheme for a group of unmanned aerial vehicles to simultaneously observe multiple concentration levels of an atmospheric plume. The authors incorporated the estimations of the concentration into the uncertainty at these levels to find out the optimal sampling locations. Wu et al. [76] proposed a switching scheme for a team of mobile sensors to switch between individual exploration and cooperative exploration as they were exploring an unknown environment. It is proposed that the density field is defined 
by mapping uncertainty, it changes with every measurement taken; a symmetry-preserving coordinated motion strategy for sensing agents was delineated in [77] to provide optimal measurements.

Cortés in [78] developed a distributed Kriged Kalman filter for robotic wireless sensors to predict the field of interest. A consensus algorithm is implemented on new measurements to calculate state predictions of the field. A gradient based controller was designed to drive the mobile wireless sensors to take optimal samples so that the variance of the estimate error is decreased. In [79], Oh et al. proposed a distributed learning algorithm for robotic sensing systems, called cross validation. In this proposition, each mobile sensor learns model parameters using its own measurements and sends the learned parameters to other sensors to validate until all mobile sensors share the best fitness results.

$\mathrm{Xu}$ et al. primarily used the GP regression for estimating and predicting the generally scalar field and designed optimality criteria based on the Fisher information matrix [80] and the average of the prediction error variances [81], [82] for the optimal sampling paths of the MRWSNs. A maximum likelihood recursive filter is proposed to learn unknown model parameters for the covariance function as well as the basis functions. More specially, in [82], a theoretical foundation of the GP regression with a subset of measurements is derived for the MRWSNs. Based on this proposition, a gradient descent based algorithm is delineated to manage the sensing robot coordination. The authors in [83] introduced the Bayesian optimization based technique for the purpose of choosing the much more relevant informative locations for the MRWSs in the GP modelled field. Moreover, a new utility function based on travelled distances of the MRWSs was also proposed to be used indirectly in trade-off between the exploration and the exploitation of the mobile sensors. In our previous works [84], [85], efficient approaches based on the conditional entropy and posterior variances were proposed to design near-optimal sampling paths for the mobile robotic sensors, where it is more important that the solutions were proved to be bound.

\section{GAUSSIAN MARKOV RANDOM FIELD FOR SENSOR PLACEMENT}

With respect to the GP model, the computational issues have always been a bottleneck, since the computational complexity of factorizing dense covariance matrices is cubic in dimension, which is known as "the big n problem" [86]. In the context of statistics, this challenge has been dealt with by a reducedrank approximation of the Gram matrix [87], a sparse greedy approach [88], and a sparse GP [89]. Recently, [82] proposed an approach to diminish the computational complexity of a large dense covariance matrix by shortening mobile sensor observations. The disadvantage of this method is that the model parameters need to be known in advance. Other efforts are to represent a continuously spatial process by a discretely indexed Gaussian field. In other words, [90]-[93] have attempted to enhance the computational complexity in modelling the spatial field by replacing the GP by a computationally efficient
Gaussian Markov random field (GMRF) [94]. The GMRF is considerably specified by a sparse precision matrix that makes it substantially advantageous to effective computation. The sparsity property of the precision matrix is constituted by a conditional independence concept in which conditional distribution of every random variable only depends on its neighbours. As a consequence, the sparsity of the precision matrix allows the GMRF to have received more and more attention for resource-constrained MRWSNs as compared to the standard GP [74], [95]-[99]. In their work [74], Ny et al. introduced an estimator by the use of the Kalman filter, where the sensor trajectory optimization problem based upon an information criterion is in turn a deterministic optimal control problem. In addition, [95] represented an approach in which the physical phenomenon of interest is regularly discretized and modelled by a GMRF. In this proposition, the hyperparameters of the GMRF model are supposed to be known and chosen with a support. The authors in [98] introduced a new class of a GP built on a GMRF for modelling the spatial process. Nonetheless, this technique proposes a model with a known precision matrix. More interestingly, Jadaliha et al. [96] investigated the GMRF to tackle the simultaneous localization and spatial prediction problem in a fully Bayesian fashion. These authors also considered localization uncertainty of the mobile sensing agent in the spatial prediction utilizing the GMRF in another work [97]. However, the approaches proposed by the works [95]-[98] are limited to a representation of a regular lattice, which requires the model parameters to be known a priori.

\section{Conclusions}

In this paper, a detailed literature review on the spatial prediction by the utilization of the WSNs has been given. Various criteria for the sensor selection problem in the SWSNs have been proposed, which have been then addressed by different approaches, in the literature. Many other methods to resolve centralized and distributed spatial prediction issues and to find the sampling paths for the MRWSs in the sensor placement problems have been introduced. Some disadvantages of the existing techniques have been also analysed.

\section{REFERENCES}

[1] M. P. Pata, T. Kneib, C. Cadarso-Suárez, V. Lustres-Pérez, and E. Fernández-Pulpeiro, "Categorical structured additive regression for assessing habitat suitability in the spatial distribution of mussel seed abundance," Environmetrics, vol. 23, pp. 75-84, 2012.

[2] M. Francisco-Fernández, A. Q. del Río, and R. Fernández-Casal, "Nonparametric methods for spatial regression. An application to seismic events," Environmetrics, vol. 23, pp. 85-93, 2012.

[3] Z. Zhu and M. L. Stein, "Spatial sampling design for prediction with estimated parameters," Journal of Agricultural, Biological, and Environmental Statistics, vol. 11, pp. 24-44, 2006.

[4] T. Harmon, C. Kvien, D. Mulla, G. Hoggenboom, J. Judy, and J. Hook, "Precision agriculture scenario," in Proc. NSF Workshop on Sensors for Environmental Observatories, Baltimore, MD, USA, 2005.

[5] P. J. Diggle and P. J. Ribeiro, Model-based geostatistics. Springer, New York, USA, 2007.

[6] N. D. Le and J. V. Zidek, Statistical analysis of environmental spacetime processes. Springer, New York, 2006. 
[7] L. V. Nguyen, S. Kodagoda, R. Ranasinghe, and G. Dissanayake, "Simulated annealing based approach for near-optimal sensor selection in gaussian processes," in Proc. IEEE International Conference on Control, Automation and Information Science, Ho Chi Minh city, Vietnam, November 2012, pp. 142-147.

[8] L. V. Nguyen and S. Kodagoda, "Soil organic matter estimation in precision agriculture using wireless sensor networks," in Proc. 2016 14th IEEE International Conference on Control, Automation, Robotics and Vision, Phuket, Thailand, November 2016, pp. 1-6.

[9] L. V. Nguyen, G. Hu, and C. J. Spanos, "Spatio-temporal environmental monitoring for smart buildings," in Proc. IEEE International Conference on Control and Automation, Ohrid, Macedonia, July 2017, pp. 277-282.

[10] H. T. Nguyen, L. V. Nguyen, and H. X. Le, "Efficient approach for maximizing lifespan in wireless sensor networks by using mobile sinks," ETRI Journal, vol. 39(3), pp. 353-363, 2017.

[11] S. Boyd and L. Vandenberghe, Convex optimization. Cambridge University Press, 2004.

[12] F. Pukelsheim, Optimal design of experiments. Society for Industrial and Applied Mathematics, 2006.

[13] K. Chaloner and I. Verdinelli, "Bayesian experimental design: A review," Statistical Science, vol. 10(3), pp. 273-304, 1995.

[14] P. Müller, B. Sanso, and M. D. Iorio, "Optimal Bayesian design by inhomogeneous Markov chain simulation," Journal of the American Statistical Association, vol. 99(467), pp. 788-798, 2004.

[15] N. Nguyen and A. J. Miller, "A review of some exchange algorithms for constructing discrete D-optimal designs," Computational Statistics and Data Analysis, vol. 14(4), pp. 489-498, 1992.

[16] S. Joshi and S. Boyd, "Sensor selection via convex optimization," IEEE Transaction on Signal Processing, vol. 57, pp. 451-462, 2009.

[17] M. Naeem, U. Pareek, and D. C. Lee, "Swarm intelligence for sensor selection problems," IEEE Sensors Journal, vol. 12(8), pp. 2577-2585, 2012.

[18] F. Ghassemi and V. Krishnamurthy, "Separable approximation for solving the sensor subset selection problem," IEEE Transaction on Aorospace and Electronic Systems, vol. 47, pp. 557-568, 2011.

[19] S. B. Crary and Y. Jeong, "Bayesian optimal design of experiments for sensor calibration," in Proc. International Conference on Solid-State Sensors and Actuators, and Eurosensors IX, Stockholm, Sweden, 1995, pp. $48-51$

[20] A. Krause, A. Singh, and C. Guestrin, "Near-optimal sensor placements in Gaussian processes: Theory, efficient algorithm and empirical studies," Journal of Machine Learning Research, vol. 9, pp. 235-284, 2008.

[21] L. V. Nguyen, S. Kodagoda, and R. Ranasinghe, "Spatial sensor selection via Gaussian Markov random fields," IEEE Transaction on Systems, Man, and Cybernetics: Systems, vol. 46(9), pp. 1226-1239, 2016.

[22] H. Choi, J. P. How, and P. I. Barton, "An outer-approximation approach for information-maximizing sensor selection," Optimization Letter, vol. 7, pp. 745-764, 2013.

[23] J. Yun and J. Kim, "Deployment support for sensor networks in indoor climate monitoring," International Journal of Distributed Sensor Networks, pp. 1-10, 2013.

[24] M. S. Uddin, A. Kuh, A. Kavcic, and T. Tanaka, "Nested performance bounds and approximate solutions for the sensor placement problem," APSIPA Transactions on Signal and Information Processing, vol. 3, pp. $1-13,2014$

[25] W. Welch, "Branch and bound search for experimental designs based on D optimality and other criteria," Technometrics, vol. 24, pp. 41-48, 1982.

[26] C. Ko, J. Lee, and M. Queyranne, "An exact algorithm for maximum entropy sampling," Operations Research, vol. 43, pp. 684-691, 1995.

[27] F. Bian, D. Kempe, and R. Govindan, "Utility based sensor selection," in Proc. 17th International Conference on Information Processing Sensor Networks, Tennessee, USA, April 2006, pp. 11-18.

[28] S. Kirkpatric, C. D. Gelatt, and M. P. Vecchi, "Optimization by simulated annealing," Science, vol. 220, pp. 671-680, 1983.

[29] V. Gupta, T. Chung, B. Hassibi, and R. Murray, "On a stochastic sensor selection algorithm with applications in sensor scheduling and sensor coverage," Automatica, vol. 42(2), pp. 251-260, 2006.

[30] P. Sebastiani and H. P. Wynn, "Maximum entropy sampling and optimal bayesian experimental design," Journal of the Royal Statistical Society, vol. 62, pp. 145-157, 2000

[31] L. Paninski, "Asymptotic theory of information-theoretic experimenta design," Neural Computation, vol. 17, pp. 1480-1507, 2005.

[32] Z. Zainudin, S. Kodagoda, and L. V. Nguyen, "Mutual information based data selection in gaussian processes for people tracking," in Proc.
Australian Conference on Robotics and Automation, Wellington, New Zealand, December 2012

[33] N. A. Cressie, Statistics for spatial data. Wiley, 1991.

[34] W. F. Caselton and J. V. Zidek, "Optimal monitoring network designs," Statistics and Probability Letters, vol. 2, pp. 223-227, 1984.

[35] L. V. Nguyen, R. Ranasinghe, S. Kodagoda, and G. Dissanayake, "Sensor selection based routing for monitoring gaussian processes modeled spatial phenomena," in Proc. Australian Conference on Robotics and Automation, Wellington, New Zealand, December 2012.

[36] C. Guestrin, A. Krause, and A. P. Singh, "Near-optimal sensor placements in Gaussian processes," in Proc. 22th International Conference on Machine Learning, Bonn, Germany, 2005, pp. 265-272.

[37] L. V. Nguyen, S. Kodagoda, R. Ranasinghe, G. Dissanayake, H. Bustamante, D. Vitanage, and T. Nguyen, "Spatially-distributed prediction with mobile robotic wireless sensor networks," in Proc. 2014 13th IEEE International Conference on Control, Automation, Robotics and Vision, Marina Bay Sands, Singapore, December 2014, pp. 1130-1135.

[38] B. Korte and J. Vygen, Combinatorial optimization: Theory and algorithms. Springer, 2012.

[39] L. V. Nguyen, G. Hu, and C. J. Spanos, "Efficient spatio-temporal sensor deployments: A smart building application," in Proc. IEEE International Conference on Control and Automation, Ohrid, Macedonia, July 2017, pp. 612-617.

[40] L. Yao, W. A. Sethares, and D. C. Kammer, "Sensor placement for onorbit modal identification via a genetic algorithm," AIAA, vol. 31, pp. 1922-1928, 1993.

[41] B. Suman and P. Kumar, "A survey of simulated annealing as a tool for single and multiobjective optimization," Operational Research Society, vol. 57, pp. 1143-1160, 2006.

[42] J. P. Sheu, K. Y. Hsieh, and P. W. Cheng, "Design and implementation of mobile robot for nodes replacement in wireless sensor networks," Journal of Information Science and Engineering, vol. 24, pp. 393-410, 2008.

[43] M. D. Francesco, S. K. Das, and G. Anastasi, "Data collection in wireless sensor networks with mobile elements: A survey," ACM Transactions on Sensor Networks, vol. 8(1), pp. 7:1-7:31, 2011.

[44] Q. Dong and W. Dargie, "A survey on mobility and mobility-aware MAC protocols in wireless sensor networks," IEEE Communications Surveys and Tutorials, vol. 15(1), pp. 88-100, 2013.

[45] R. C. Shah, S. Roy, S. Jain, and W. Brunette, "Data MULEs: modeling a three-tier architecture for sparse sensor networks," in Proc. IEEE International Workshop on Sensor Network Protocols and Applications, Anchorage, AK, USA, May 2003, pp. 30-41.

[46] C. H. Wu, W. Sheng, and Y. Zhang, "Mobile sensor networks self localization based on multi-dimensional scaling," in Proc. IEEE International Conferece on Robotics and Automation, Rome, Italy, April 2007, pp. 4038-4043

[47] S. Gao, H. Zhang, and S. Das, "Efficient data collection in wireless sensor networks with path-constrained mobile sinks," in Proc. IEEE International Symposium on a World of Wireless, Mobile and Multimedia Networks and Workshops, Kos, Greece, June 2009, pp. 1-9.

[48] O. Tekdas, V. Isler, J. H. Lim, and A. Terzis, "Using mobile robots to harvest data from sensor fields," IEEE Wireless Communications, vol. 16(1), pp. 22-28, 2009.

[49] T. C. Chen, T. S. Chen, and P. W. Wu, "On data collection using mobile robot in wireless sensor networks," IEEE Transactions on Systems, Man, and Cybernetics - Part A: Systems, vol. 41(6), pp. 1213-1224, 2011.

[50] L. He, J. Pan, and J. Xu, "An on-demand data collection scheme for wireless sensor networks with mobile elements," in Proc. IEEE International Conference on Communications, Kyoto, Japan, June 2011, pp. 1-5.

[51] G. Fletcher, L. Xu, A. Nayak, and I. Stojmenovic, "Randomized robotassisted relocation of sensors for coverage repair in wireless sensor networks," in Proc. IEEE Vehicular Technology Conference, Ottawa, Canada, September 2010, pp. 1-5.

[52] L. Xu, R. Falcon, A. Nayak, and I. Stojmenovic, "Servicing wireless sensor networks by mobile robots," IEEE Communications Magazine, vol. 50(7), pp. 147-154, 2012.

[53] C. Tang and P. McKinley, "Energy optimization under informed mobility," IEEE Transactions on Parallel and Distributed Systems, vol. 17(9), pp. $947-962,2006$

[54] G. Dini, M. Pelagatti, and I. M. Savino, "An algorithm for reconnecting wireless sensor network partitions," in Proc. 5th European conference on Wireless Sensor Networks, Bologna, Italy, January 2008, p. 253267. 
[55] Y. Mei, C. Xian, S. Das, Y. C. Hu, and Y. H. Lu, "Replacing failed sensor nodes by mobile robots," in Proc. IEEE International Conference on Distributed Computing Systems, Workshop on Wireless Ad hoc and Sensor Networks, Lisboa, Portugal, July 2006, pp. 1 - 6.

[56] K. A. Luthy, E. Grant, and T. C. Henderson, "Leveraging RSSI for robotic repair of disconnected wireless sensor networks," in Proc. IEEE International Conferece on Robotics and Automation, Rome, Italy, April 2007, pp. 3659 - 3664

[57] J. Cortes, S. Martínez, T. Karatas, and F. Bullo, "Coverage control for mobile sensing networks," IEEE Transactions on Robotics and Automation, vol. 20, pp. 243-254, 2004.

[58] L. V. Nguyen, S. Kodagoda, R. Ranasinghe, and G. Dissanayake, "Locational optimization based sensor placement for monitoring gaussian processes modeled spatial phenomena," in Proc. 8th IEEE International Conference on Industrial Electronics and Applications, Melbourne, Australia, June 2013, pp. 1706-1711.

[59] N. E. Leonard, D. Paley, F. Lekien, R. Sepulchre, D. M. Fratantoni, and R. Davis, "Collective motion, sensor networks and ocean sampling," Proceedings of the IEEE, vol. 95(1), pp. 48-74, 2007.

[60] S. Martínez, "Distributed interpolation schemes for field estimation by mobile sensor networks," IEEE Transactions on Control Systems Technology, vol. 18, pp. 491-500, 2010.

[61] S. Huang and J. Tan, "Adaptive sampling using mobile sensor networks," in Proc. IEEE International Conferece on Robotics and Automation, Minnesota, USA, May 2012, pp. 657-662.

[62] J. Binney, A. Krause, and G. S. Sukhatme, "Optimizing waypoints for monitoring spatiotemporal phenomena," The International of Robotics Research, vol. 32(8), pp. 873-888, 2013.

[63] R. Ouyang, K. H. Low, J. Chen, and P. Jaillet, "Multi-robot active sensing of non-stationay Gaussian process-based environmental phanomena," in Proc. International Foundation for Autonomous Agents and Multiagent Systems, Paris, France, 2014, pp. 573-580.

[64] H. M. La, W. Sheng, and J. Chen, "Cooperative and active sensing in mobile sensor networks for scalar field mapping," in Proc. IEEE International Conference on Automation Science and Engineering, WI, USA, 2013, pp. 831-836.

[65] L. V. Nguyen, S. Kodagoda, R. Ranasinghe, and G. Dissanayake, "Spatially-distributed prediction with mobile robotic wireless sensor networks," in Proc. 2014 13th IEEE International Conference on Control, Automation, Robotics and Vision, Marina Bay Sands, Singapore, December 2014, pp. 1153-1158.

[66] M. Jadaliha and J. Choi, "Environmental monitoring using autonomous aquatic robots: Sampling algorithms and experiments," IEEE Transactions on Control Systems Technology, vol. 21, pp. 899-905, 2013.

[67] J. Suh and S. Oh, "Efficient environmental monitoring using cost-aware path planning," in Proc. 13th International Conference on Control, Automation and Systems, Gwangju, Korea, 2013, pp. 1362-1365.

[68] R. Graham and J. Cortes, "A cooperative deployment strategy for optima sampling in spatiotemporal estimation," in Proc. IEEE Conference on Decision and Control, Cancun, Mexico, 2008, pp. 2432-2437.

[69] _ _ "Distributed sampling of random fields with unknown covariance," in Proc. American Control Conference, MI, USA, 2009, pp. 4543-4548.

[70] — - "Cooperative adaptive sampling via approximate entropy maximization," in Proc. IEEE Conference on Decision and Control, Shanghai, China, 2009, pp. 7055-7060.

[71] D. O. Popa, M. F. Mysorewala, and F. L. Lewis, "EKF-based adaptive sampling with mobile robotic sensor nodes," in Proc. IEEE/RSJ International Conferece on Intelligent Robots and Systems, Beijing, China, October 2006, pp. 2451-2456.

[72] — - "Adaptive sampling using non-linear EKF with mobile robotic wireless sensor nodes," in Proc. IEEE International Conference on Control, Automation, Robotics and Vision, Grand Hyatt, Singapore, December 2006, pp. 1-6.

[73] J. Choi, J. Lee, and S. Oh, "Swarm intelligence for achieving the global maximum using spatio-temporal Gaussian processes," in Proc. American Control Conference, Washington, USA, June 2008, pp. 135-140.

[74] J. L. Ny and G. J. Pappas, "On trajectory optimization for active sensing in Gaussian process models," in Proc. Joint 48th IEEE Conference on Decision and Control and 28th Chinese Control Conference, Shanghai, China, 2009, pp. 6286-6292.

[75] J. Euler, A. Horn, D. Haumann, J. Adamy, and O. V. Stryk, "Cooperative n-boundary tracking in large scale environments," in Proc. IEEE International Conference on Mobile Adhoc and Sensor Systems, Las Vegas, NV, USA, October 2012, pp. 1-6.
[76] W. Wu and F. Zhang, "Robust cooperative exploration with a switching strategy," IEEE Transactions on Robotics, vol. 28(4), pp. 828 - 839 2012.

[77] C. H. C.-N. nez and N. E. Leonard, "Symmetric coverage of dynamic mapping error for mobile sensor networks," in Proc. IEEE American Control Conference, San Francisco, CA, USA, 2011, pp. 4661-4666.

[78] J. Cortés, "Distributed Kriged Kalman filter for spatial estimation," IEEE Transactions on Automatic Control, vol. 54, pp. 2816-2827, 2009.

[79] S. Oh and J. Choi, "Distributed learning in mobile sensor networks using cross validation," in Proc. 49th IEEE International Conference on Decision and Control, Atlanta, USA, 2010, pp. 3845-3850.

[80] Y. Xu and J. Choi, "Adaptive sampling for learning Gaussian processes using mobile sensor networks," Sensors, vol. 11, pp. 3051-3066, 2011.

[81] Y. Xu, J. Choi, S. Dass, and T. Maiti, "Sequential Bayesian prediction and adaptive sampling algorithms for mobile sensor networks," IEEE Transactions on Automatic Control, vol. 57, pp. 2078-2084, 2012.

[82] Y. Xu, J. Choi, and S. Oh, "Mobile sensor network navigation using Gaussian processes with truncated observations," IEEE Transactions on Robotics, vol. 27, pp. 1118-1131, 2011

[83] R. Marchant and F. Ramos, "Bayesian optimization for intelligent environmental monitoring," in Proc. IEEE/RSJ International Conferece on Intelligent Robots and Systems, Algarve, Portugal, October 2012, pp. 2242-2249.

[84] L. V. Nguyen, S. Kodagoda, R. Ranasinghe, and G. Dissanayake, "Information-driven adaptive sampling strategy for mobile robotic wireless sensor network," IEEE Transaction on Control Systems Technology, vol. 24(1), pp. 372-379, 2016.

[85] — "Adaptive placement for mobile sensors in spatial prediction under locational errors," IEEE Sensors Journal, vol. 17(3), pp. 794 - 802, 2017.

[86] S. Banerjee, B. Carlin, and A. Gelfand, Hierarchical modeling and analysis for spatial data. Chapman and Hall, New York, 2004.

[87] C. K. I. Williams and M. Seeger, "Using the Nyström method to speed up kernel machines," Advances in Neural Information Processing Systems, vol. 13, 2001.

[88] A. J. Smola and P. Bartlett, "Sparse greedy Gaussian process regression," Advances in Neural Information Processing Systems, vol. 13, 2001.

[89] N. Lawrence, M. Seeger, and R. Herbrich, "Fast sparse Gaussian process methods: The informative vector machine," in Proc. 16th Annual Conference on Neural Information Processing Systems, Vancouver, BC , USA, 2003, pp. 609-616.

[90] H. Rue and H. Tjelmeland, "Fitting Gaussian Markov random fields to Gaussian fields," Scandinavian Journal of Statistics, vol. 29, pp. 31-49, 2002.

[91] N. Cressie and N. Verzelen, "Conditional-mean least-squares fitting of Gaussian Markov random fields to Guassian fields," Computational Statistics and Data Analysis, vol. 52, pp. 2794-2807, 2008.

[92] L. Hartman and O. Hössjer, "Fast kriging of large data sets with Gaussian Markov random fields," Computational Statistics and Data Analysis, vol. 52, pp. 2331-2349, 2008.

[93] F. Lindgren, H. Rue, and J. Lindström, "An explicit link between Gaussian fields and Gaussian Markov random fields: The stochastic partial differential equation approach," Journal of the Royal Statistical Society (Series B), vol. 73, pp. 423-498, 2011.

[94] H. Rue and L. Held, Gaussian Markov random field: Theory and applications. Chapman and Hall - CRC Press, London, England, 2005.

[95] Y. Xu, J. Choi, S. Dass, and R. Maiti, "Efficient Bayesian spatial prediction with mobile sensor networks using Gaussian Markov random fields," Automatica, vol. 49, pp. 3520-3530, 2013.

[96] M. Jadaliha and J. Choi, "Fully Bayesian simultaneous localization and spatial prediction using Gaussian Markov random fields,' in Proc. American Control Conference, Washington DC, USA, June 2013, pp. 4599-4604.

[97] M. Jadaliha, Y. Xu, and J. Choi, "Efficient spatial prediction using Gaussian Markov random fields under uncertain localization," in Proc. ASME Dynamic Systems and Control Conference, Florida, USA, October 2012.

[98] Y. Xu and J. Choi, "Spatial prediction with mobile sensor networks using Gaussian processes with built-in Gaussian Markov random fields," Automatica, vol. 48, pp. 1735-1740, 2012.

[99] L. V. Nguyen, S. Kodagoda, R. Ranasinghe, and G. Dissanayake, "Mobile robotic wireless sensor networks for efficient spatial prediction," in Proc. 2014 IEEE/RSJ International Conference on Intelligent Robots and Systems, Chicago, IL, USA, September 2014, pp. 1176-1181. 